\title{
Topological Design of Planar Circularly Polarized Directional Antenna with Low Profile Using Particle Swarm Optimization
}

\author{
Xiaonan Zhao, ${ }^{1,2}$ Junping Geng, ${ }^{1}$ Ronghong Jin, ${ }^{1}$ Yao Jin, ${ }^{1}$ Xiang Liu, ${ }^{1}$ and Wenyan Yin ${ }^{1}$ \\ ${ }^{1}$ Department of Electronics Engineering, Shanghai Jiao Tong University, Shanghai 200240, China \\ ${ }^{2}$ China Ship Development and Design Center, Wuhan, China \\ Correspondence should be addressed to Xiaonan Zhao; sherlock_zxn@hotmail.com
}

Received 29 January 2017; Accepted 11 April 2017; Published 17 May 2017

Academic Editor: Davide Ramaccia

Copyright (c) 2017 Xiaonan Zhao et al. This is an open access article distributed under the Creative Commons Attribution License, which permits unrestricted use, distribution, and reproduction in any medium, provided the original work is properly cited.

\begin{abstract}
A topological method for the design and optimization of planar circularly polarized (CP) directional antenna with low profile was presented. By inserting two parasitic layers, generated by particle swarm optimization, between the equiangular spiral antenna and the ground, a low-profile wideband CP antenna with directional radiation pattern and high gain is achieved. The optimized antenna shows an impedance matching band $\left(\left|S_{11}\right|<-10 \mathrm{~dB}\right)$ of $4-12 \mathrm{GHz}$ with a whole-band stable directional pattern in $4-11.5 \mathrm{GHz}$, and the antenna gain peak is $8 \mathrm{dBi}$, which work well in the available band. Measured return loss, antenna gain, and far field patterns agree well with simulation results.
\end{abstract}

\section{Introduction}

Planar circularly polarized (CP) antennas are widely used in modern wireless communications owing to their compact sizes $[1,2]$ that can be flexibly integrated with various systems. For achieving good directionality and high gain, a ground plane is often used at $1 / 4$ wavelength away from the planar antenna as a reflector [3], which results in the deterioration of the frequency bandwidths of planar antennas and limits their scope of applications. At the motivations of increasing demands for different characteristics including high gain, wide impedance band, and low fabrication cost, many smart improvements are made based on original planar antennas [4]. For examples, photonic bandgap (PBG) [5] or electromagnetic band gap (EBG) structures [6] can be used for reducing the profiles of spiral antennas. A UWB spiral antenna with parabolic reflector was designed in [7], where VSWR $<2$ was achieved in a wide band from $0.94 \mathrm{GHz}$ to $4.27 \mathrm{GHz}$. A small disc backed by a cavity [8] was introduced under a single-arm spiral antenna and the periphery of the cavity was loaded using ring-shaped absorbing material to realize an extremely low-profile wideband CP directional antenna. Corrugated reflectors are adopted to keep the ultrawideband characteristic with a stable radiation pattern [9].
In [10], an irregular ground plane consisting of a circular part and several extended curved branches is proposed for a circular patch; the antenna and the irregular ground plane are shortened by a set of conductive vias to achieve wideband impedance matching.

These ingenious designs rely much on designers' skill and experience. To offer an easy method for designing and optimizing low-profile $\mathrm{CP}$ directional antennas, we proposed a universal scheme along with a discrete meshing optimization method to generate two optimized 2D parasitic layers, which acts as a metamaterial between the existed equiangular spiral antenna and the ground plane to achieve good radiation performance.

\section{Semiautomatic Optimization Method}

Inspired by the principle of metamaterial, which owns smart properties capable of blocking, absorbing, enhancing, or bending electromagnetic waves, we tried to find a common way to generate a metamaterial structure automatically for the optimization of planar antennas.

Firstly, the optimized antenna includes multitargets, such as lower profile, better $\mathrm{CP}$ characteristic, higher gain, and 


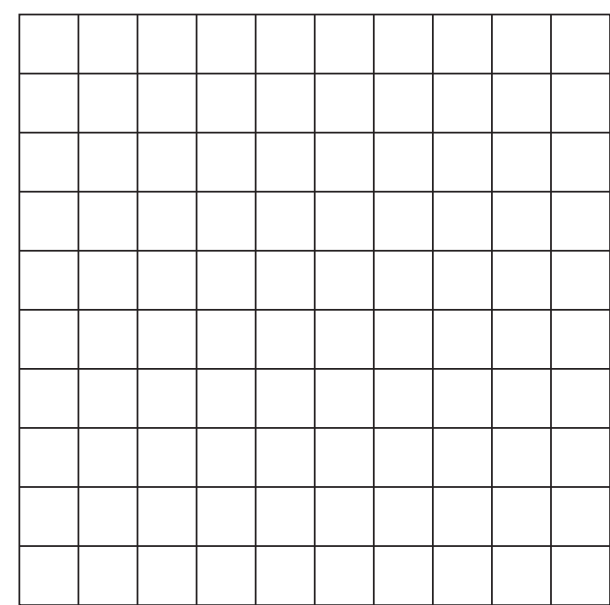

(a)

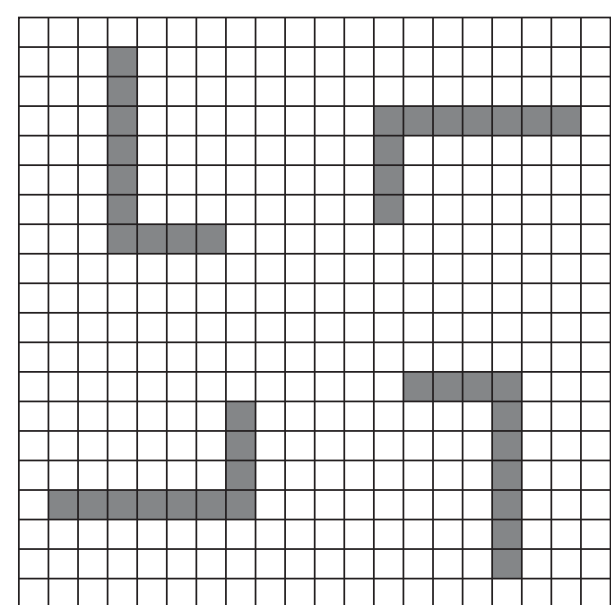

(b)

FIgURE 1: (a) Discrete grid model and (b) the rotational symmetrical layer.

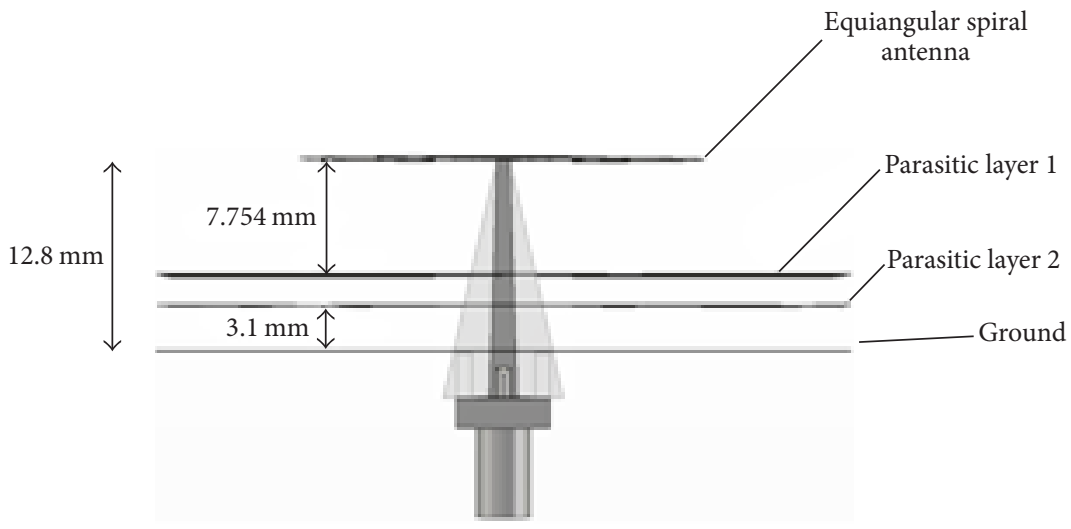

FIGURE 2: Side view of the basic scheme.

wider frequency band, so that a thin (for low profile) rotational symmetrical structure (for CP beam) with reflectance bigger than 0 (for high gain in wideband) is set as the design direction. We proposed a rotational symmetrical grid parasitic layer between the planar antenna and its ground as a preliminary plan. To make it easier to get good "metamaterial" characteristic, we took two parallel layers with different discrete grid pattern and named them parasitic layers. Each parasitic layer is equally divided into four parts, and one of them is meshed. The mesh should not be too coarse, which would make it hard for optimization, and should not be too refined, which would not be convenient for fabrication. Refer to the mesh size in computational electromagnetics (CEM) which is always set as one-tenth of the wavelength; we mesh each parasitic layer's first quarter part into $10 \times 10$ grids, as shown in Figure 1(a). The remaining three quarter parts are rotational symmetrical to the first one with 90-degree angle difference, as shown in Figure 1(b).

Optimization algorithm is used to determine whether each grid of the $1 / 4$ part is to be filled with metal sheet or left empty, while the rest three quarter parts are rotational symmetrical same counter parts of the first quarter part. Thus the energy would be assembled to the center, which is somewhat like bending electromagnetic waves. Both parasitic layers are designed in this way.

In order to prove parasitic layers' effect as a metamaterial and their efficiency in antenna improvement, we took an example.

\section{Example of an Equiangular Spiral Antenna}

3.1. Optimized Antenna. We take an equiangular spiral antenna as an example to make this process more detailed. The initial structure of the antenna structure is suggested in [11]. A microstrip-to-balanced stripline balun [4] is used as the feeding, which is shown in Figure 2. Then the two rectangle parasitic layers with same size as the antenna's ground are introduced. Both of them have a circular hole in the center for the feeding.

There are many optimization algorithms commonly used in electromagnetics including antenna design, such as genetic 


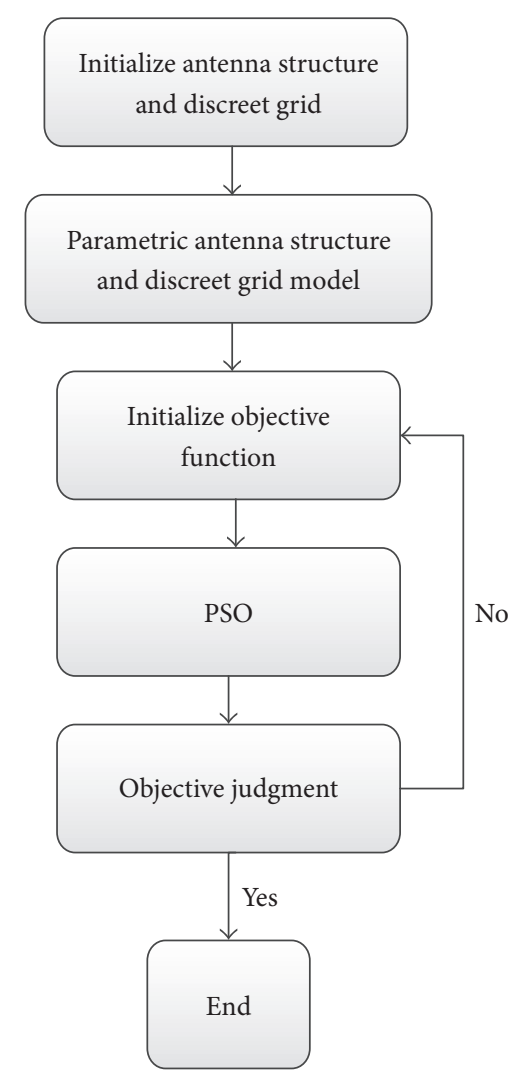

FIGURE 3: Antenna optimization process.

algorithm [12, 13], particle swarm optimization (PSO) [14$17]$, and more recent biogeography-based optimization [18, 19].

Here, we choose PSO as the optimization algorithm. The antenna optimization process is shown in Figure 3.

For the antenna structure, the optimization is based on continuous variables. For the parasitic layers with grid structure, optimization concerns discrete variable, which has only two values, denoted with or without grid. In order to unify the discrete and continuous variables into the same particle swarm optimization (PSO) and single fitness function, we used an improved mixed-integer multiobjective PSO. By consulting [10], PSO was edited by Visual Basic and then added to CST Microwave Studio through the macro. The detailed process is as follows. Different from [13], we define the grid element code height $i(j, k)=0.9 \sim 1.1$, where "int $(i(j, k))=1$ " represents the grid with metal if $i(j, k) \geqslant 1$ and "int $(i(j, k))=0$ " represents the grid without metal if $i(j, k)<1$. The code height is similar to the length parameter of every grid and can be regarded as particles similar to other parameters in the PSO model in CST or any other commercial EM software.

The optimized targets (OT) include $\left|S_{11}\right|$, wideband (wb), directivity, and gain $(G)$ in the available band, and the target function can be described as

$$
\mathrm{OT}=w_{1} \times\left|S_{11}\right|+\frac{w_{2}}{\mathrm{wb}}+\frac{w_{3}}{G\left(\theta<30^{\circ}\right)} .
$$

Here, $w_{1}, w_{2}$, and $w_{3}$ are the weights of the targets and $G\left(\theta<30^{\circ}\right)$ is the combined target of directivity and gain, which means the gain when the main beam of the antenna is in $\theta<30^{\circ}$. Usually, we set $w_{1}=w_{2}=$ $w_{3}=1.0$, which can be adjusted after the crude good results appeared.

With the above optimization process, the parameters of the equiangular spiral antenna structure and the parasitic layers can be optimized at the same time.

After optimization, the top layer is an equiangular spiral antenna as shown in Figure 4(a) and the two parasitic layers are shown in Figures 4(b) and 4(c), respectively.

With $H=12.8 \mathrm{~mm}, L=44.2 \mathrm{~mm}$, and the maximum radius of the equiangular spiral antenna, $13.75 \mathrm{~mm}$, this semiautomatic designed antenna has a low-profile structure, which is suitable to be installed on plane platform.

3.2. Transmission and Reflection Performance of the Optimized Parasitic Layers. In order to verify the "metamaterial" characteristic of the optimized parasitic layers in concerned frequencies, we simulated the transmission and reflection performance from $3 \mathrm{GHz}$ to $12 \mathrm{GHz}$.

Here, two cases are set for simulation. In Case 1, the $\mathrm{CP}$ plane wave just radiated to the ground, as shown in Figure 5(a). Reflected wave power flow density at the parallel probe plane A-A (which is $\lambda / 2$ above the ground) and transmitted wave power flow density at the parallel probe 


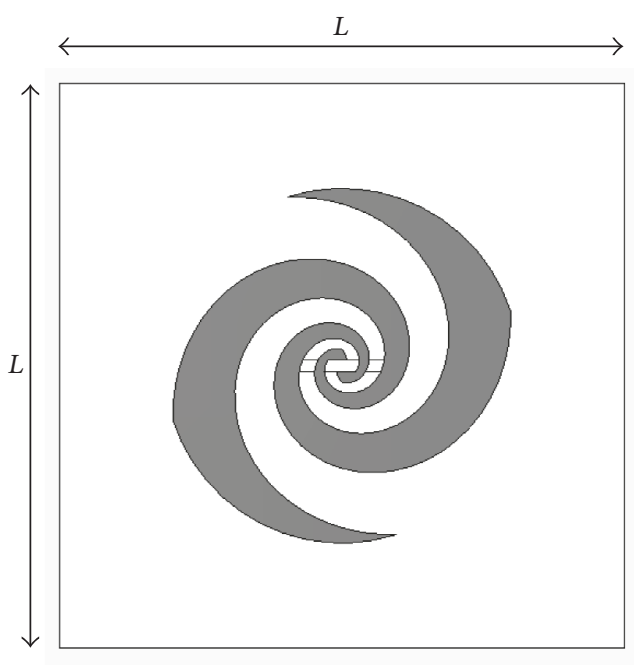

(a)

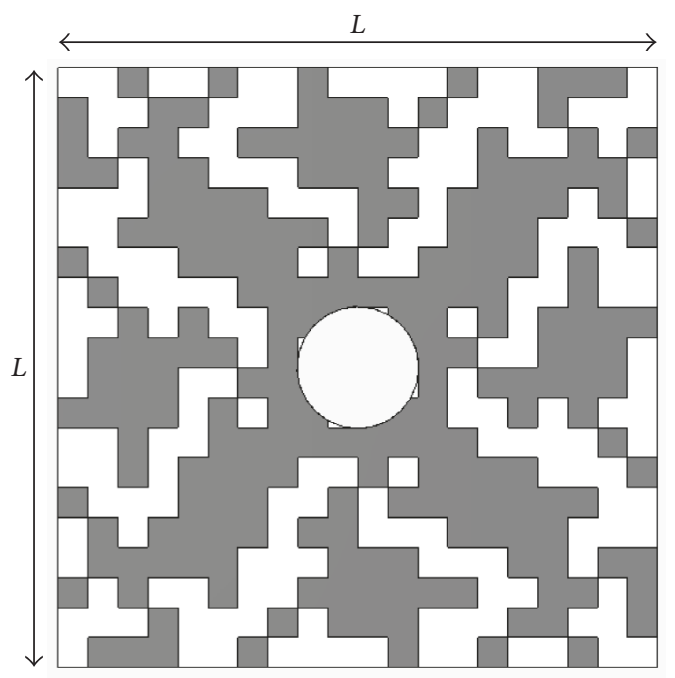

(c)

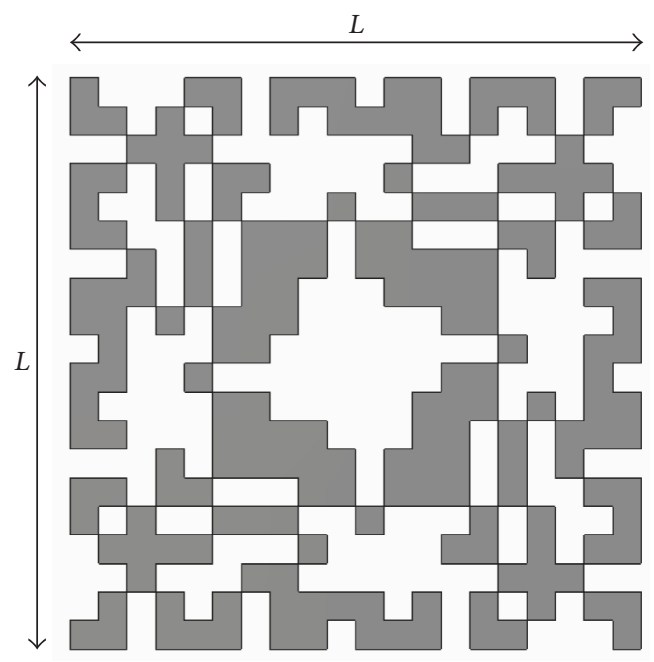

(b)

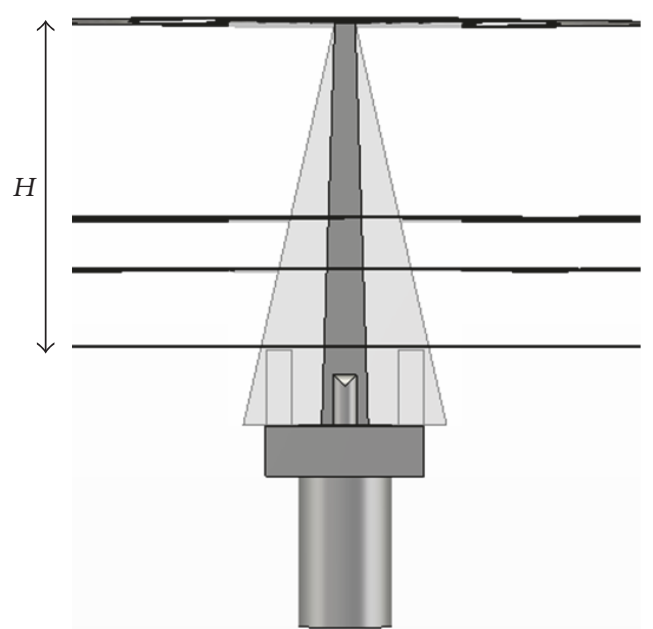

(d)

FIgURE 4: Antenna structure: (a) equiangular spiral antenna, (b) parasitic layer 1, (c) parasitic layer 2, and (d) side view and balun.

plane B-B (which is $\lambda / 2$ below the ground) are simulated; results at typical frequencies are shown in Figure 5.

In Figure 5(b), the reflected power flow density distribution is very close to the transmitted power flow at low frequencies and becomes larger than the transmitted power flow at higher frequencies. But the reflected power flow density distribution is very different from the transmitted power flow.

In Case 2, the $\mathrm{CP}$ plane wave radiates through the two optimized parasitic layers with ground, as shown in Figure 6(a). For the same samples, $f=4 \mathrm{GHz}, 6 \mathrm{GHz}$, and $8 \mathrm{GHz}$; the reflected power flow density is much more stronger than that in Case 1. It is obvious that adding the parasitic layers would strengthen and homogenize the reflected wave power flow density and meanwhile reduce the transmitted wave power flow density.
Further, we discretized the same-sized probe plane into $40 \times 40$ grids. The total reflected or transmitted wave power is calculated in Table 1 . The data shows that inserting the two parasitic layers could transfer the fixed narrow-band ground with -1 reflection into a wideband "metamaterial" structure with positive reflection.

Graphical comparisons are shown in Figures 7 and 8. In Figure 7, the upper parasitic layer shows stronger reflected wave power in low frequency band than the lower parasitic layer and the same in the middle band but smaller in the higher band, so that the total reflected power from the two parasitic layers keeps stronger in $4 \mathrm{GHz} \sim 9 \mathrm{GHz}$.

In Figure 8, the upper parasitic layer transmitted less power in lower frequency band but increased quickly in higher band. The lower parasitic layer transmitted small power and equilibrium in the whole band, so that the total 


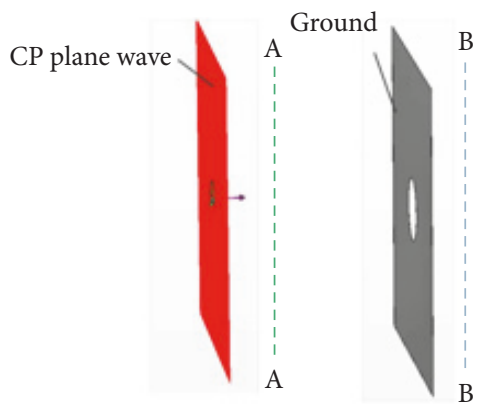

(a)

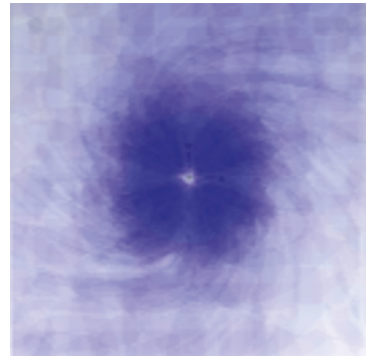

(b)

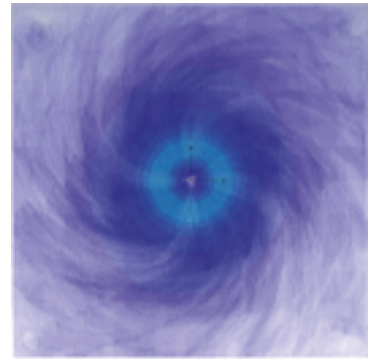

(d)

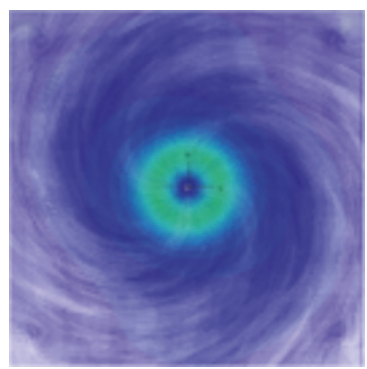

(f)

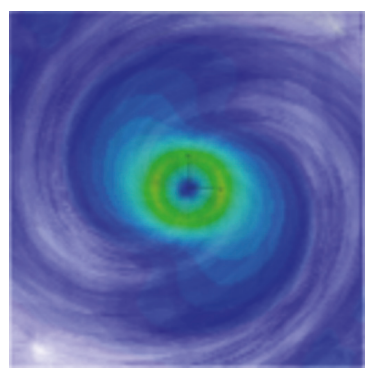

(h)

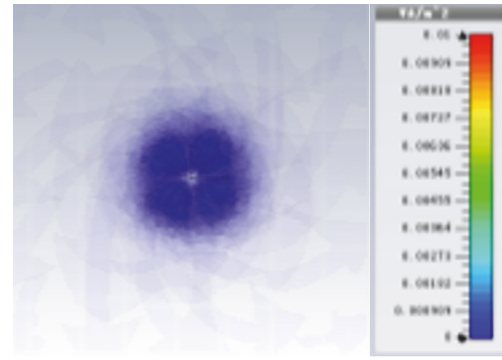

(c)

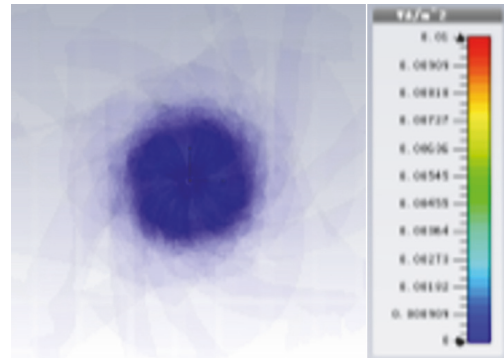

(e)

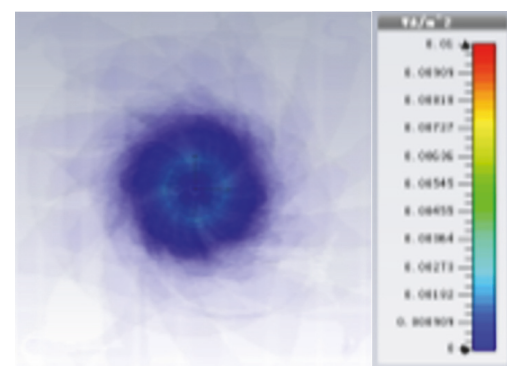

(g)

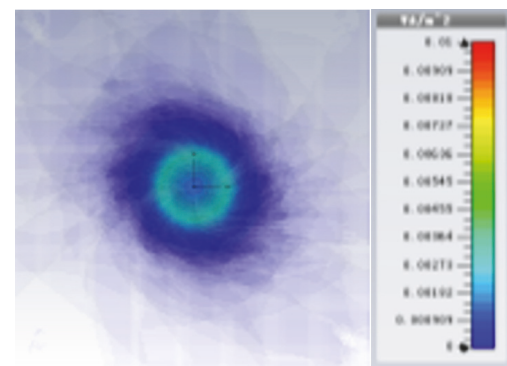

(i)

FIGURE 5: Case 1: (a) circularly polarized plane wave radiates on the limited ground. Simulated reflected wave power flow density at (b) $4 \mathrm{GHz}$, (d) $6 \mathrm{GHz}$, (f) $8 \mathrm{GHz}$, and (h) $10 \mathrm{GHz}$. Simulated transmitted wave power flow density at (c) $4 \mathrm{GHz}$, (e) $6 \mathrm{GHz}$, (g) $8 \mathrm{GHz}$, and (i) $10 \mathrm{GHz}$. 


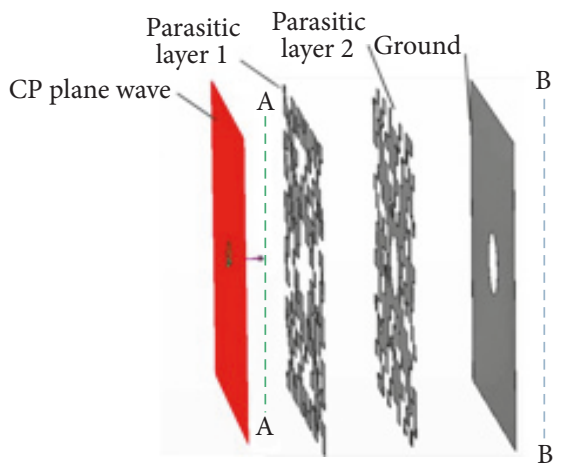

(a)

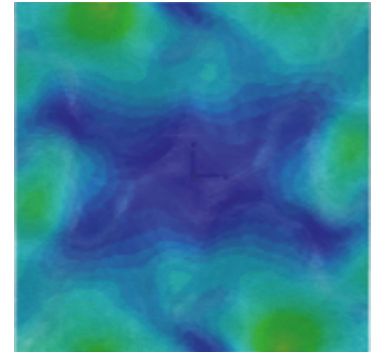

(b)

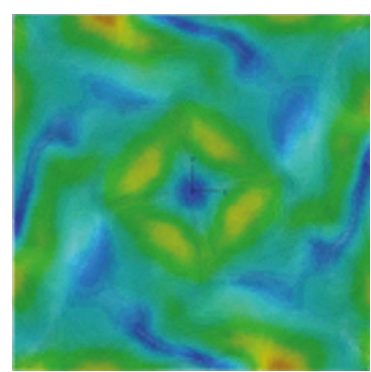

(d)

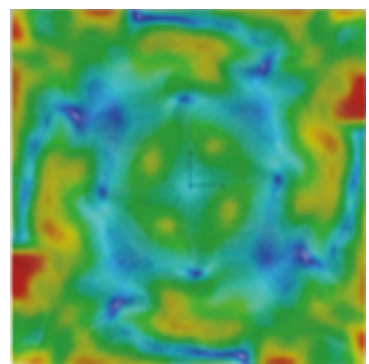

(f)

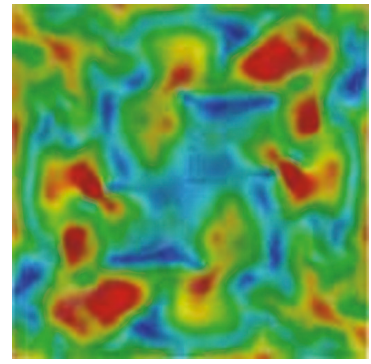

(h)

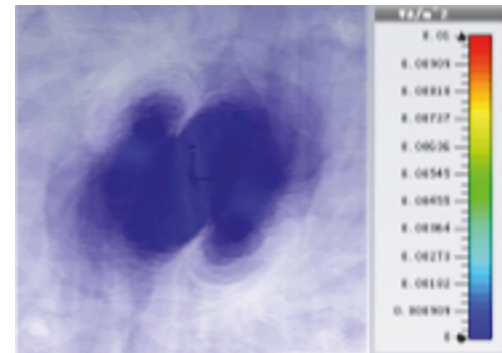

(c)

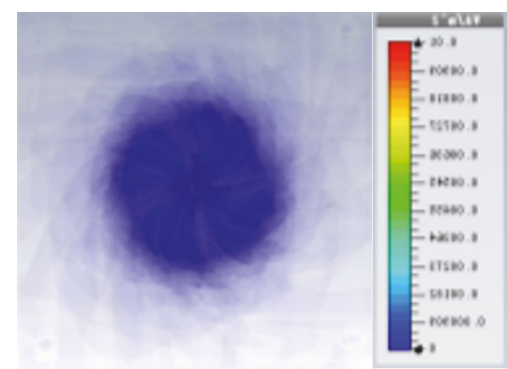

(e)

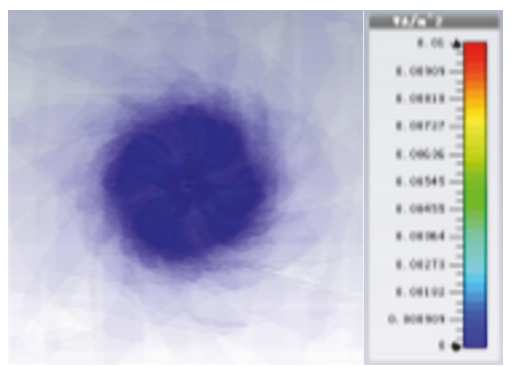

(g)

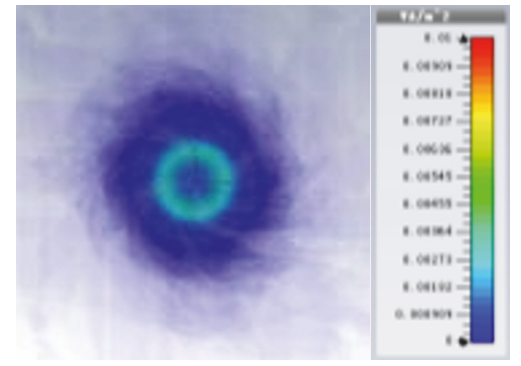

(i)

FIGURE 6: Case 2: (a) circularly polarized plane wave radiates on the limited ground. Simulated reflected wave power flow density at (b) $4 \mathrm{GHz}$, (d) $6 \mathrm{GHz}$, (f) $8 \mathrm{GHz}$, and (h) $10 \mathrm{GHz}$. Simulated transmitted wave power flow density at (c) $4 \mathrm{GHz}$, (e) $6 \mathrm{GHz}$, (g) $8 \mathrm{GHz}$, and (i) $10 \mathrm{GHz}$. 


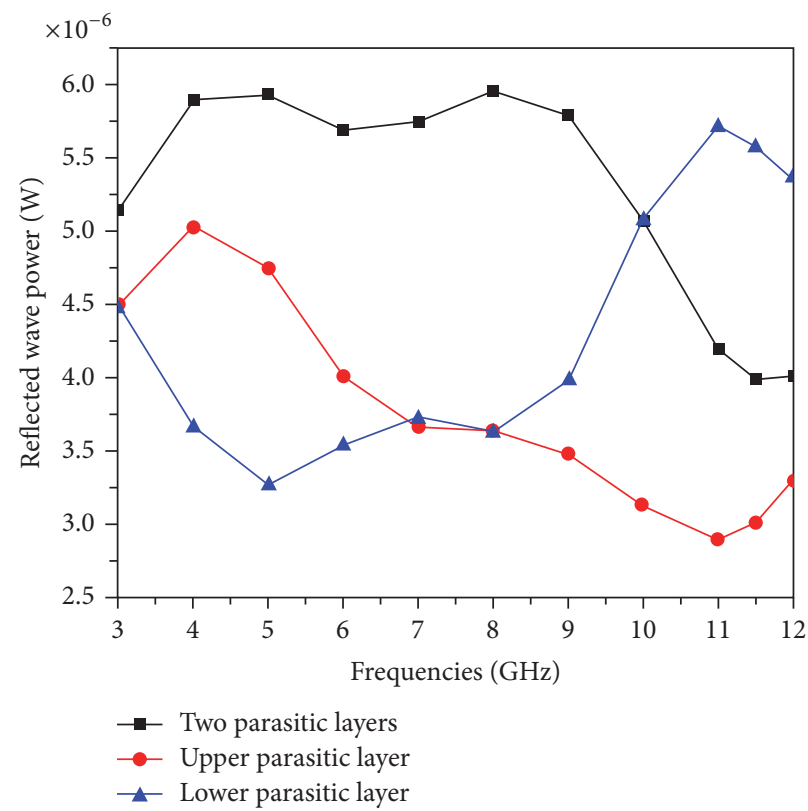

FIGURE 7: Total reflected wave power flow for parasitic layers alone and together.

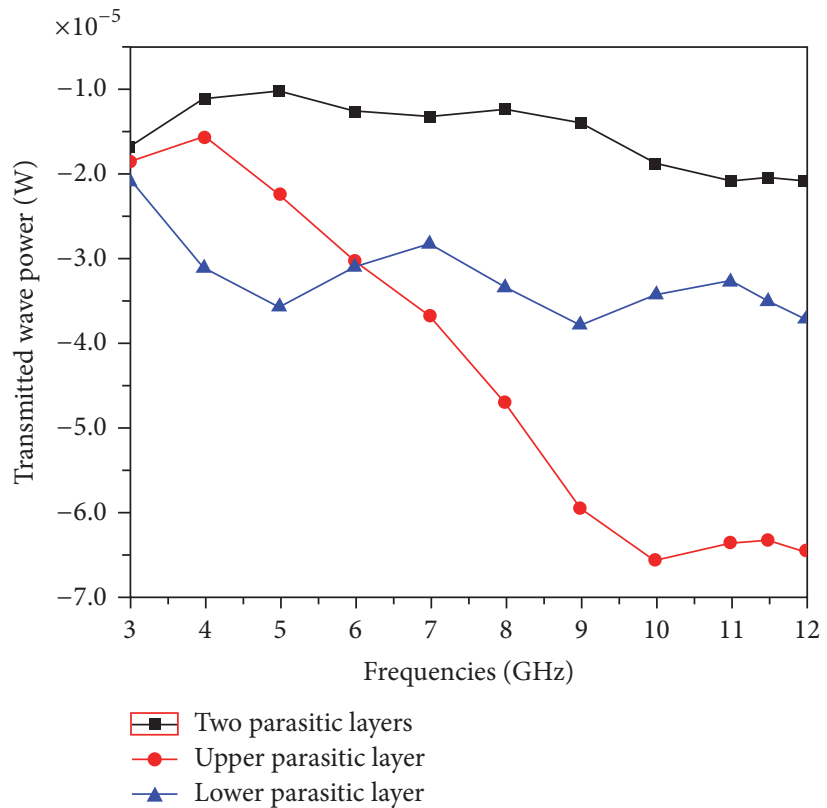

FIGURE 8: Total transmitted wave power flow for parasitic layers alone and together.

transmitted power from these two parasitic layers is very small.

Figures 7 and 8 clearly reveal the frequency selective characteristics of single quasi-periodic parasitic layer in reflection and transmission.

So, combining the two parasitic layers and ground, just as in Figure 5(a), the incident plane wave in lower frequency firstly is reflected by the upper parasitic layer, and the higher frequency band wave is mainly transmitted to the lower parasitic layer and then reflected. Only few left waves reach the ground and would be reflected back to the parasitic layer again.

It is clear that the combination of the different frequency selective parasitic layers and the ground would reflect the wave back in very wide band.

\section{Results Analysis}

We marked the equiangular spiral antenna with ground only as Antl, in which the distance between equiangular spiral 
TABLE 1: Total reflected and transmitted wave power flow for different structure (normalization value).

\begin{tabular}{lccccccccccccc}
\hline \multirow{2}{*}{ Analyzed structures } & Component & & \multicolumn{1}{c}{ Frequencies $(\mathrm{GHz})$} \\
& & 3 & 4 & 5 & 6 & 7 & 8 & 9 & 10 & 11 & 11.5 & 12 \\
\hline \multirow{2}{*}{ None } & Trans & 1 & 1 & 1 & 1 & 1 & 1 & 1 & 1 & 1 & 1 & 1 \\
& Ref & 0 & 0 & 0 & 0 & 0 & 0 & 0 & 0 & 0 & 0 & 0 \\
\hline \multirow{2}{*}{ Case 1 } & Trans & 0 & 0 & 0 & 0 & 0 & 0 & 0.01 & 0.01 & 0.02 & 0.02 & 0.02 \\
& Ref & -1 & -1 & -1 & -1 & -1 & -1 & -1 & -1 & -1 & -1 & -1 \\
\hline \multirow{2}{*}{ Case 2 } & Trans & 0 & 0 & 0 & 0 & 0 & 0 & 0 & 0 & 0 & 0 & 0 \\
& Ref & 0.95 & 0.87 & 0.80 & 0.68 & 0.62 & 0.62 & 0.56 & 0.52 & 0.44 & 0.38 & 0.38 \\
\hline
\end{tabular}

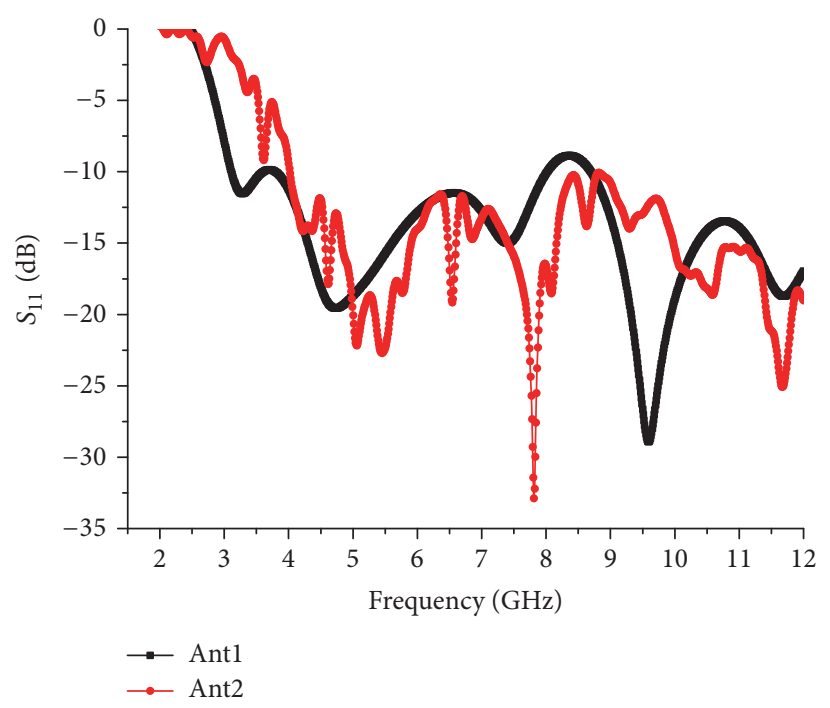

FIgure 9: Return loss for Antl and Ant2.

antenna and ground is fixed, and the bandwidth with stable directional radiation is limited. The optimized antenna is marked as Ant2 for comparison.

4.1. Simulation Results. After optimization, simulated $S_{11}$ of the two antennas is shown in Figure 9. The impedance bandwidth of Antl covers from $3 \mathrm{GHz}$ to $8 \mathrm{GHz}$ for $\left|S_{11}\right|<$ $-10 \mathrm{~dB}$, while the impedance bandwidth of Ant 2 covers from $4 \mathrm{GHz}$ to $9 \mathrm{GHz}$.

By adding the optimized parasitic layers, the far field pattern becomes more stable. Wideband and high gain are achieved simultaneously as the results shown in Figures 10 and 11.

The far field patterns of the two antennas are shown in Figure 10. For Antl without the parasitic layers, the distance from the equiangular spiral antenna to the ground is fixed, which makes the far field confusion at higher frequency, and the pattern splitted at $8 \mathrm{GHz}$. After the two parasitic layers are inserted, the "metamaterial effect" makes the max. directivity of Ant2 on different frequencies stay at the same azimuth angle; it could be said that the far field patterns of Ant 2 are more stably directional compared to Antl from $4 \mathrm{GHz}$ to $11 \mathrm{GHz}$.
The simulated gains of Ant1 and Ant2 are shown in Figure 11. From $4 \mathrm{GHz}$ to $10 \mathrm{GHz}$, the gain of Ant2 is almost higher than that of Antl.

4.2. Measurement Result. The real Ant2 is fabricated, which is $12.8 \mathrm{~mm} \times 44.2 \mathrm{~mm} \times 44.2 \mathrm{~mm}$, as shown in Figure 12 . The reflection coefficient of the fabricated antenna is shown in Figure 13. The measured impedance matching band is from $4 \mathrm{GHz}$ to $12 \mathrm{GHz}$, which is similar to the simulation result.

In Figure 14, the measured far field patterns of Ant 2 are shown at different frequencies. The measured results are similar to the simulated ones in Figure 10(b). It also shows that the far field patterns of Ant2 are stably directional.

In Figure 15, the simulated and measured gains of Ant2 are shown, which work well in most available band. The measured gain peak is about $8 \mathrm{dBi}$.

As we focus on the low-profile and directional characteristics of the antenna, which was realized by adding the metamaterial parasitic layers, and the prototype antenna is a spiral antenna, the axial ratio is not the characteristic of interest in this paper. Smaller beamwidth is another important advantage of CP antenna compared with linear 


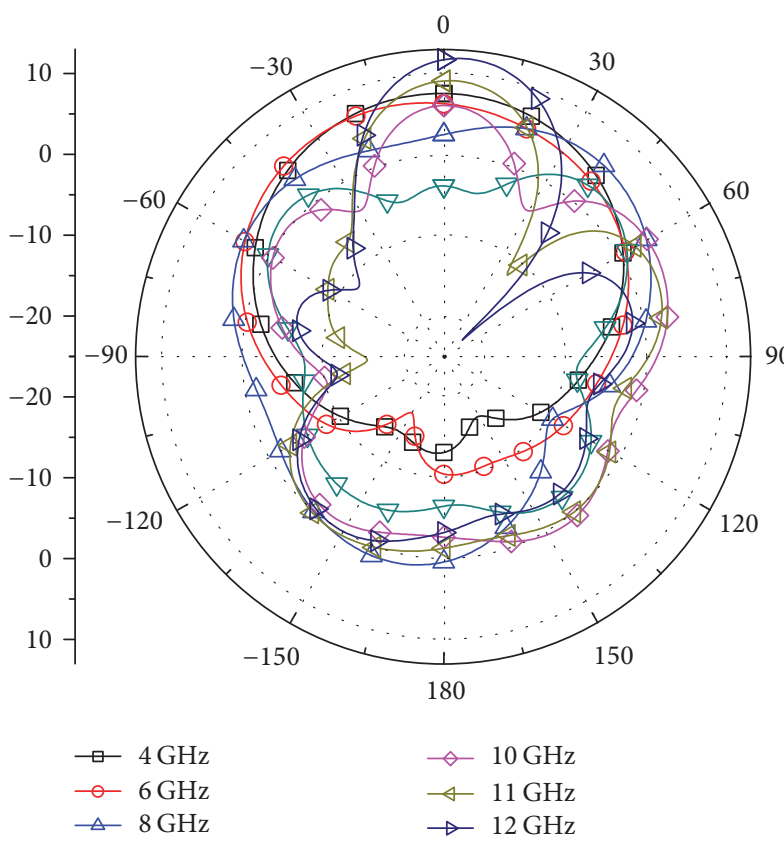

(a)

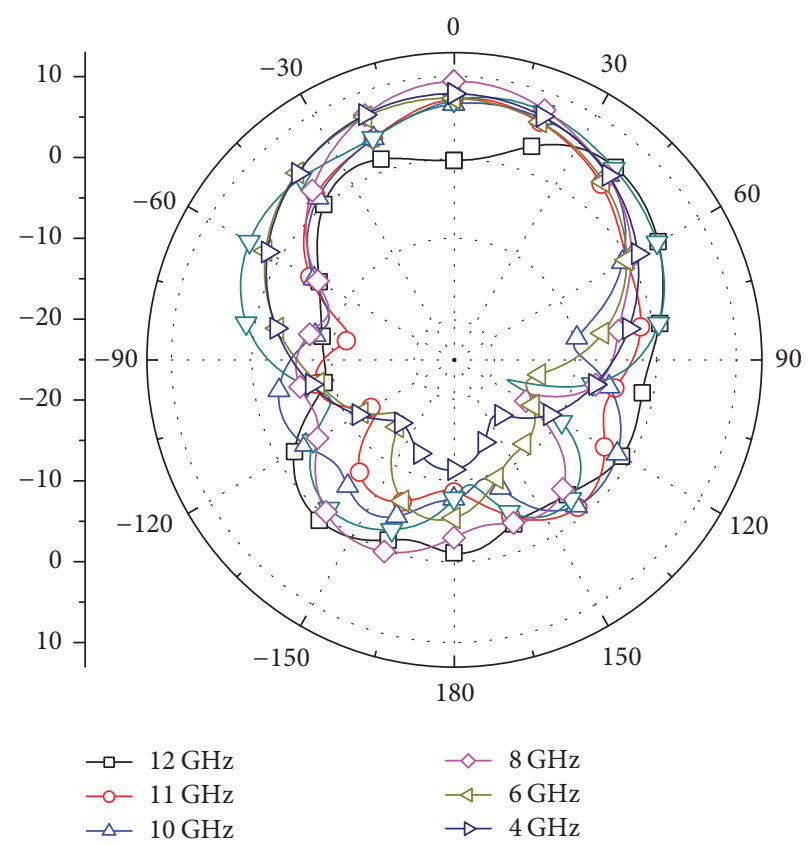

(b)

Figure 10: Far field pattern of (a) Ant1 and (b) Ant2.

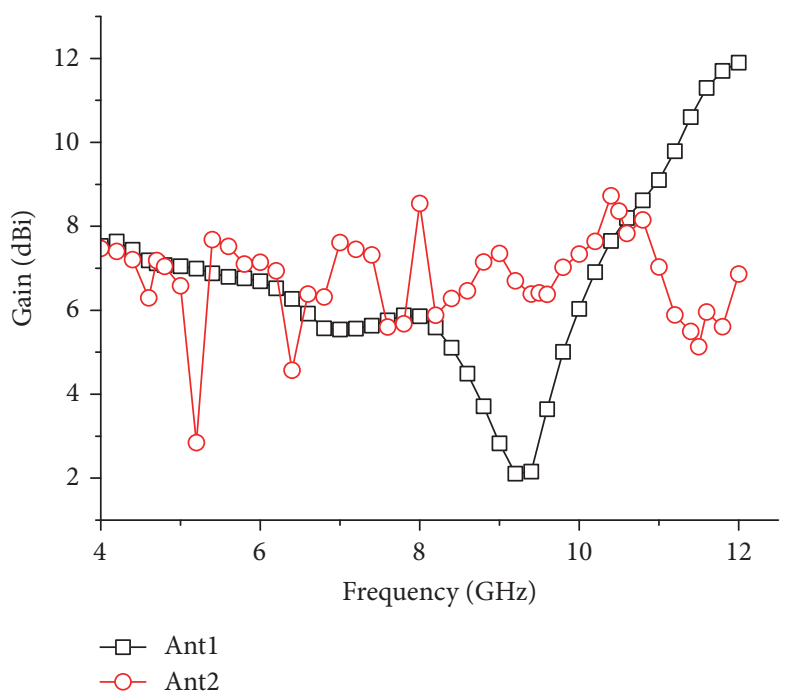

FIGURE 11: Simulated gain of Ant1 and Ant2.

polarized antenna, so the stability of the far field patterns in wideband receives more concern in this paper.

\section{Conclusion}

In this paper, we have presented a topological method for the design and optimization of planar CP directional antennas with metamaterial parasitic layers. Particle swarm optimization is employed for generating metamaterial parasitic layers. By inserting such metamaterial layers, a low-profile wideband
$\mathrm{CP}$ antenna with directional radiation pattern and high gain was designed, fabricated, and characterized. The optimized antenna has an impedance matching band $\left(\left|S_{11}\right|<-10 \mathrm{~dB}\right)$ of $4-12 \mathrm{GHz}$ with a whole-band stably directional pattern in $4-11.5 \mathrm{GHz}$, and the antenna gain peak is $8 \mathrm{dBi}$, which work well in most available band. The proposed method offers code-based self-generation of metamaterial parasitic layers, which requires less designer's skill/experience and is very suitable for improving various kinds of plane antennas with any reasonable targets. 


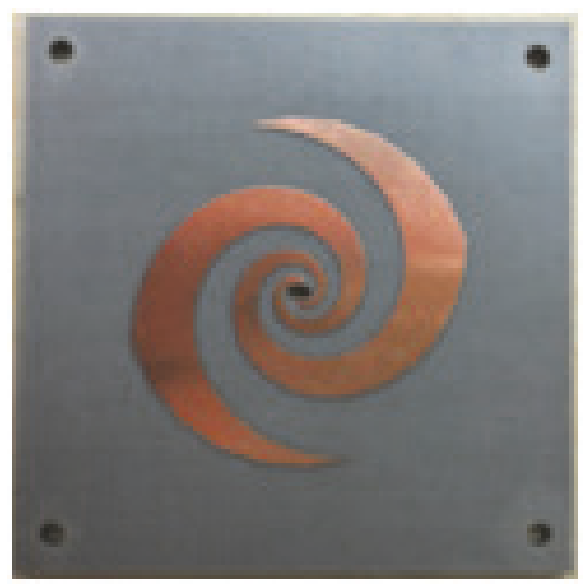

(a)

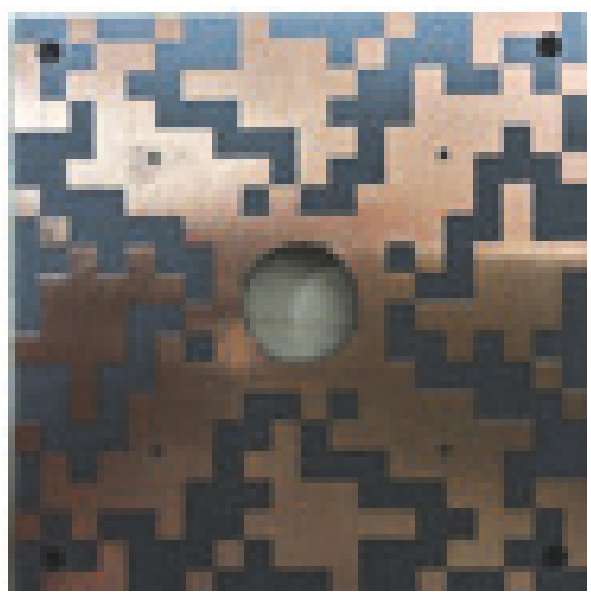

(c)

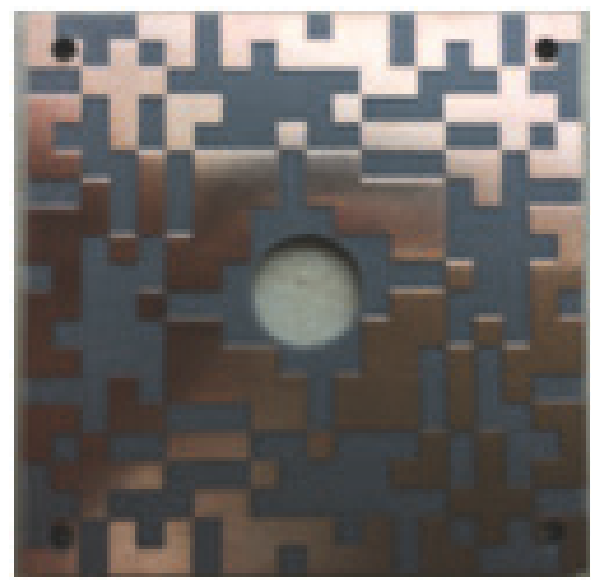

(b)

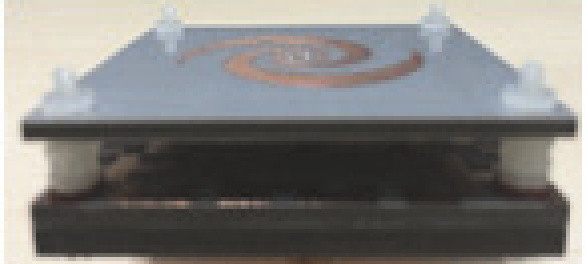

(d)

Figure 12: (a) Equiangular spiral antenna, (b) parasitic layer 1, (c) parasitic layer 2, and (d) side view of Ant2.

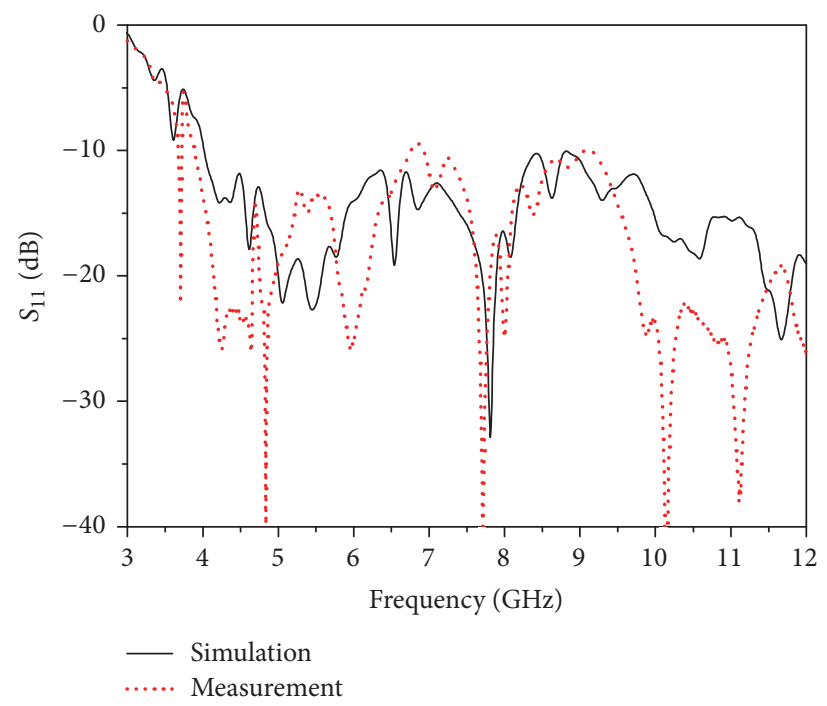

FIgURE 13: Simulated and measured $S_{11}$ of the fabricated Ant2. 


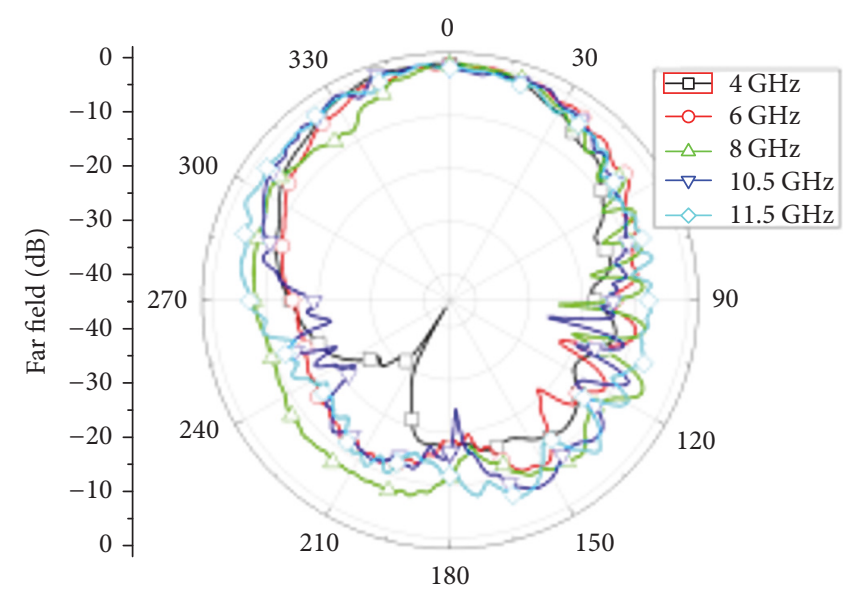

Figure 14: The measured far field pattern of Ant2 at $4 \mathrm{GHz}, 6 \mathrm{GHz}, 8 \mathrm{GHz}, 10.5 \mathrm{GHz}$, and $11.5 \mathrm{GHz}$.

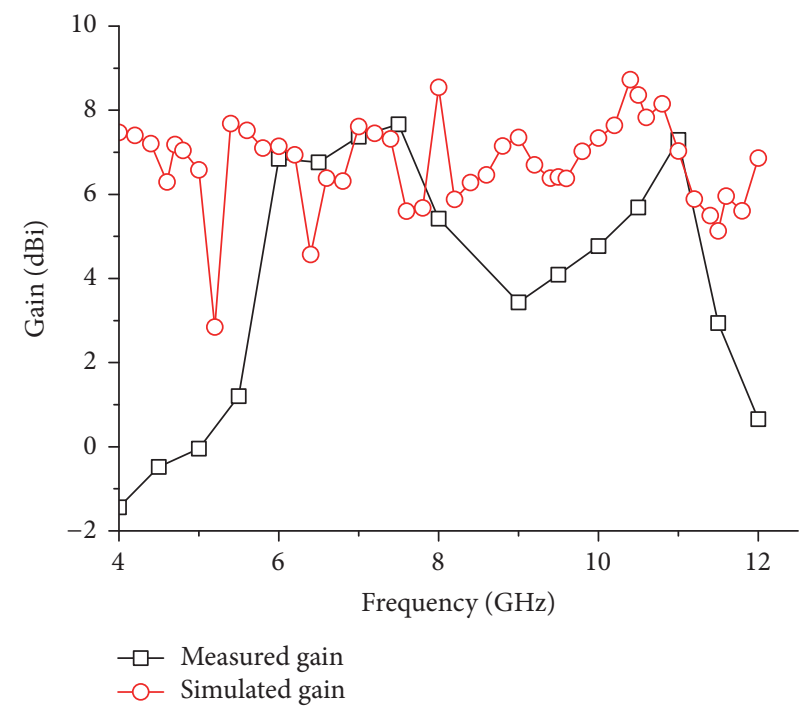

FIGURE 15: Simulated and measured gain of Ant2.

\section{Conflicts of Interest}

The authors declare that they have no conflicts of interest.

\section{References}

[1] Q. Liu, C. L. Ruan, L. Peng et al., "A novel compact archimedean spiral antenna with gap-loading," Progress In Electromagnetics Research Letters, vol. 3, pp. 169-177, 2008.

[2] K. Fujita, K. Yoshitomi, K. Yoshida, and H. Kanaya, "A circularly polarized planar antenna on flexible substrate for ultrawideband high-band applications," AEU - International Journal of Electronics and Communications, vol. 69, no. 9, pp. 1381-1386, 2015.

[3] H. Nakano, Y. Shinma, and J. Yamauchi, "A monofilar spiral antenna and its array above a ground plane-formation of a circularly polarized tilted fan beam," IEEE Transactions on Antennas and Propagation, vol. 45, no. 10, pp. 1506-1511, 1997.

[4] J. Thaysen, K. B. Jakobsen, and H.-R. Lenler-Eriksen, "Wideband cavity backed spiral antenna for stepped frequency ground penetrating radar," in Proceedings of the IEEE Antennas and Propagation Society International Symposium and USNC/URSI Meeting, pp. 418-421, July 2005.

[5] T. H. Liu, W. X. Zhang, M. Zhang, and K. F. Tsang, "Low profile spiral antenna with PBG substrate," Electronics Letters, vol. 36, no. 9, pp. 779-780, 2000.

[6] Z. Li, G. Wang, and Y. Cao, "A low-profile equiangular spiral antenna using a novel EBG ground plane," in Proceedings of the 7 th International Symposium on Antennas, Propagation \& EM Theory, (ISAPE '06), pp. 1-3, 2006.

[7] J. Lao, R. H. Jin, and J. P. Geng, "UWB spiral antenna with parabolic reflector," XXIX URSI General Assembly, Chicago, Illinois, USA, 2008, https://www.researchgate.net/publication/ 228858199_UWB_Spiral_Antenna_with_Parabolic_Reflector.

[8] H. Nakano, R. Satake, and J. Yamauchi, "Extremely low-profile, single-arm, wideband spiral antenna radiating a circularly polarized wave," IEEE Transactions on Antennas and Propagation, vol. 58, no. 5, pp. 1511-1520, 2010. 
[9] Q. Wu, R. Jin, J. Geng, and D. Su, "On the performance of printed dipole antenna with novel composite corrugatedreflectors for low-profile ultrawideband applications," IEEE Transactions on Antennas and Propagation, vol. 58, no. 12, pp. 3839-3846, 2010.

[10] Y. M. Pan, S. Y. Zheng, and B. J. Hu, "Wideband and lowprofile omnidirectional circularly polarized patch antenna," IEEE Transactions on Antennas and Propagation, vol. 62, no. 8, pp. 4347-4351, 2014.

[11] M. Ding, R. Jin, and J. Geng, "Optimal design of ultra wideband antennas using a mixed model of 2-D genetic algorithm and finite-difference time-domain," Microwave and Optical Technology Letters, vol. 49, no. 12, pp. 3177-3180, 2007.

[12] R. L. Haupt and D. H. Werner, Genetic Algorithms in Electromagnetics, Wiley-IEEE Press, 2007.

[13] Y. Kim and E. K. Walton, "Automobile conformal antenna design using non-dominated sorting genetic algorithm (NSGA)," IEE Proceedings: Microwaves, Antennas and Propagation, vol. 153, no. 6, pp. 579-582, 2006.

[14] J. Kennedy and R. Eberhart, "Particle swarm optimization," in Proceedings of the IEEE International Conference on Neural Networks (ICNN '95), vol. 4, pp. 1942-1948, 1995.

[15] J. Robinson and Y. Rahmat-Samii, "Particle swarm optimization in electromagnetics," IEEE Transactions on Antennas and Propagation, vol. 52, no. 2, pp. 397-407, 2004.

[16] N. Jin and Y. Rahmat-Samii, "Hybrid real-binary particle swarm optimization (HPSO) in engineering electromagnetics," IEEE Transactions on Antennas and Propagation, vol. 58, no. 12, pp. 3786-3794, 2010.

[17] S. Rani and A. P. Singh, "On the design and optimisation of new fractal antenna using PSO," International Journal of Electronics, vol. 100, no. 10, pp. 1383-1397, 2013.

[18] A. Sharaqa and N. Dib, "Design of linear and circular antenna arrays using biogeography based optimization," in Proceedings of the 1st IEEE Jordan Conference on Applied Electrical Engineering and Computing Technologies (AEECT '11), pp. 1-6, Amman, Jordan, December 2011.

[19] U. Singh, D. Singh, and P. Singh, "Concentric Circular Antenna Array design using hybrid differential evolution with Biogeography Based Optimization," in Proceedings of the 4th IEEE International Conference on Computational Intelligence and Computing Research, 2013. 


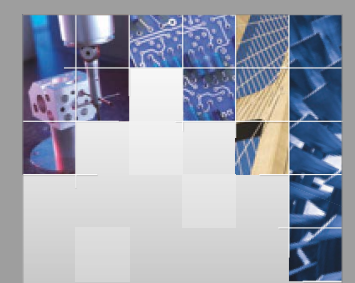

\section{Enfincering}
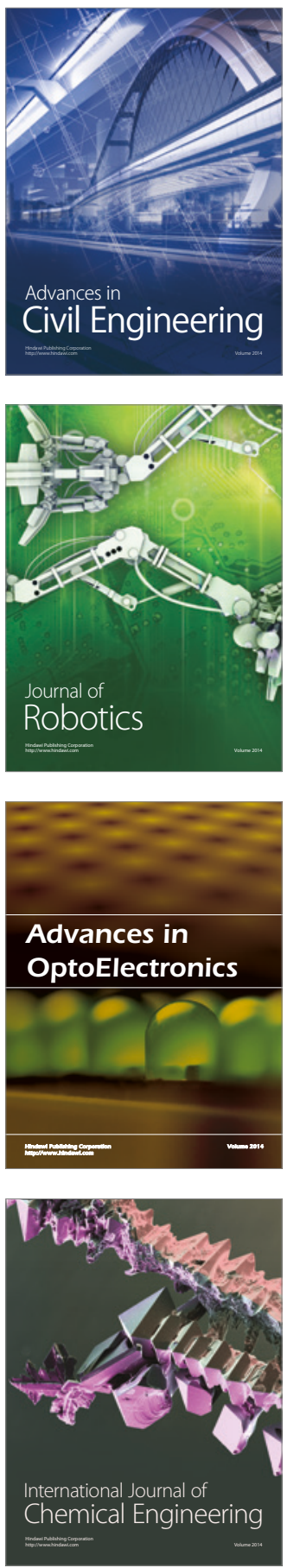

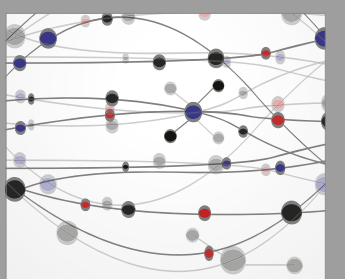

The Scientific World Journal

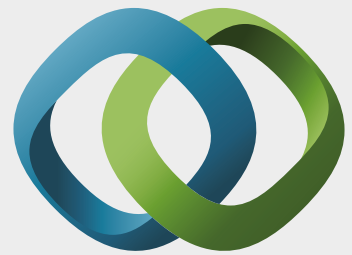

\section{Hindawi}

Submit your manuscripts at

https://www.hindawi.com
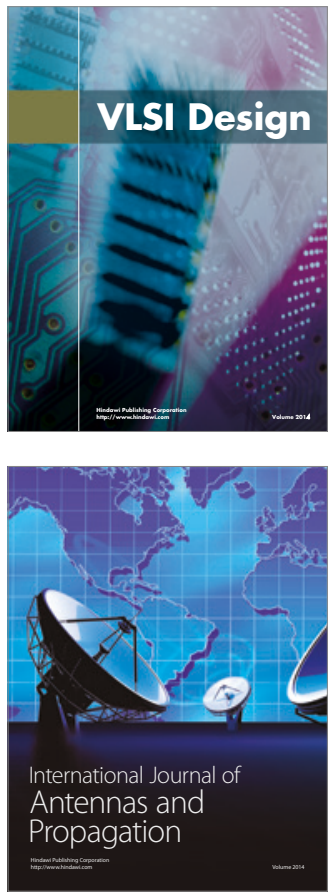

\section{Rotating}

Machinery
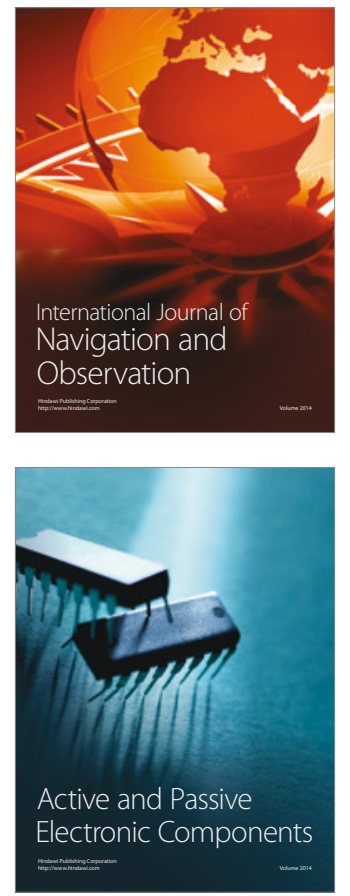
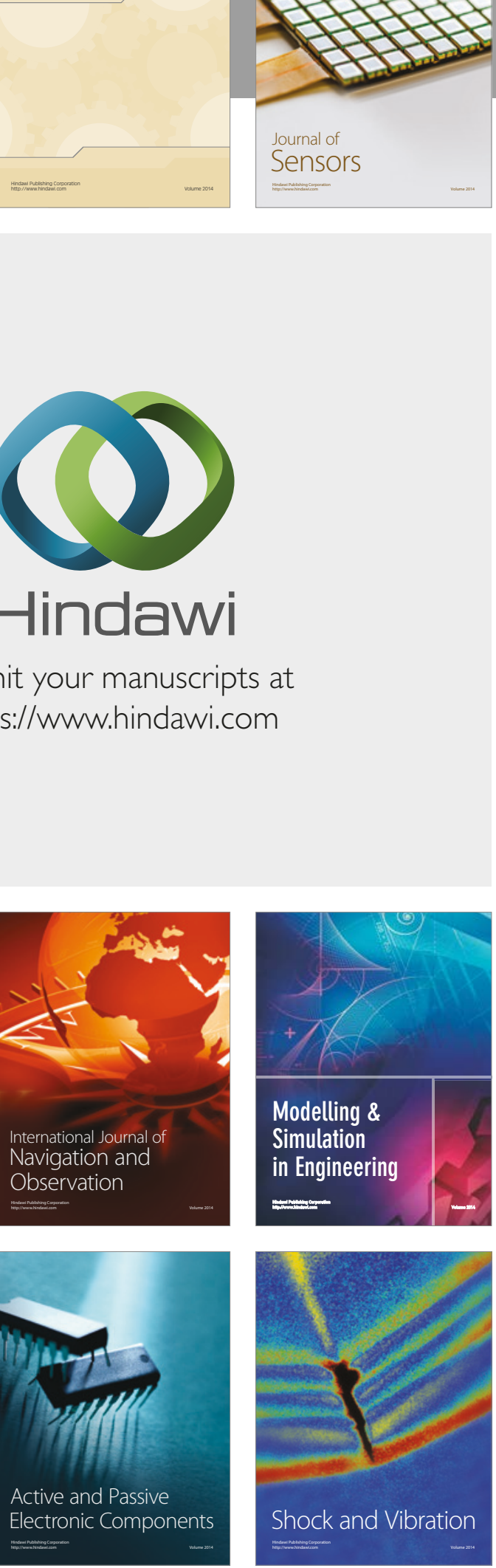
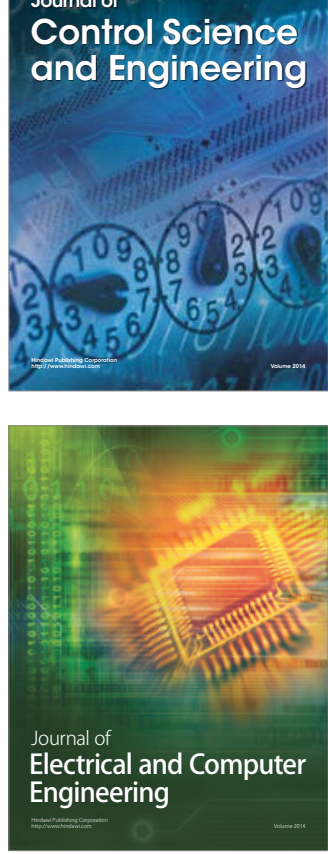

Distributed

Journal of

Control Science

and Engineering
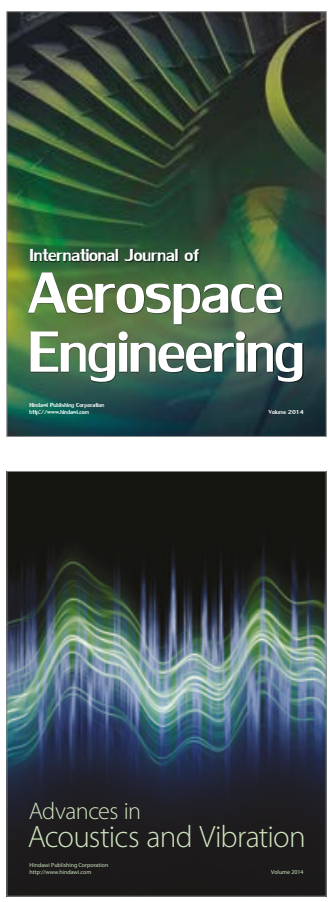

Sensor Networks 